\title{
Bildarbeit im Unterricht
}

Je weiter man sich im Geographieunterricht vom eigenen Umweltsbereich entfernt, desto mehr tritt an die Stelle der direkten Naturbeobachtung die bildhafte Anschauung als Wirklichkeitsersatz. Das Bild macht "entfernte Objekte dem Unterricht verfügbar" und erlaubt "ein Nachvollziehen des induktiven, von der Beobachtung ausgehenden Verfahrens" (4). Dadurch wird das Bild zusammen mit der Karte zum wichtigsten Unterrichtsmittel. Kein Wunder, dass heute schon eine reichhaltige didaktische Literatur vorliegt, die sich in verschiedenster Hinsicht mit dem Bild auseinandersetzt. So gibt es Arbeiten, die Massstäbe für die Beurteilung geographisch relevanter $B i l d e r$ festlegen, andere befassen sich mit der Wirkung des Bildes auf die Schuiler, mit dem stufengerechten Einsatz des Bildes, mit dem methodischen ort des Bildes innerhalb der Lektion usw. (6).

Trotzdem spricht K.E.Fick (2) von einem ernsten "Mangel an visueller Bildung und Erziehung im Erdkundeunterricht" und fährt an anderer Stelle fort: "Gelegentlich mag man sich ernsthaft und gründlich mit einem Bild befassen. Häufig bleibt das Bild aber an der Peripherie des Unterrichtes. Man überlässt es allenfalls dem Schüler, sich selbst damit auseinanderzusetzen." Gerade heute, wo wir in einem optischen Zeitalter leben und täglich einer Flut von Bildern der verschiedensten Art ausgesetzt sind, sollte die Arbeit mit dem Bild und am Bild mehr gefördert werden. Das Bild darf nicht bloss der "Veranschaulichung der Erdräume und ihrer Gegenstände dienen"(5), das heisst, begleitende Zugabe oder Illustration sein, sondern es sollte in vermehrtem Masse als Arbeitsmittel eingesetzt werden. Damit dies aber auch geschieht, benötigt der Lehrer die notwendigen Anleitungen. Diese sollten sich aber nicht auf rein theoretische Ratschläge beschränken. Wichtiger sind Unterrichtsbeispiele, die man nachvollziehen, deren Wirkung und deren Resultat man prüfen und mit den eigenen Erfahrungen vergleichen kann.

Obwohl die modernen Geographielehrbücher gegenüber früher voll. von Bildern sind, enthalten sie selten Hinweise über deren sinnvolle Verwendung im Unterricht. Nach $\mathrm{H}$. Ebeling (1) "liegt die Methodik der Bilddarbietung und -auswertung vielerorts im argen". Die Bildarbeit erfolgt nach Ansicht verschiedener Autoren oft ungeschickt und wenig überzeugend. Der planmässig vorbereitete und gezielte Umgang mit
Bildern ist leider selten, und eine richtige Anleitung zum nutzbringenden Betrachten fehlt häufig. Es leuchtet ein, dass in solchen Fällen der Bildungswert bescheiden bleibt. Die Schuiler nehmen am Gezeigten nur geringen Anteil, und damit bleibt die intensive, individuelle Beschäftigung mit dem Bildinhalt aus.

Der nachstehende, zusammenfassende Bericht über eine Bildbetrachtung will kein Beispiel, kein Modell dafür sein, wie man es macht, oder wie man es machen könnte. Die Angaben sollen vor allem den jungen Lehrer zu eigenen Versuchen, zum Experimentieren anregen. Mit voller $\mathrm{Ab}-$ sicht ist ein klar strukturiertes Bild ohne ablenkendes Beiwerk und frei von nebensächlicher Ueberfüllung gewählt worden, denn wie überall muss man auch bei der Bildarbeit mit einfachen Beispielen beginnen und erst langsam zu schwierigeren Bildinhalten übergehen. Der Zweck der Bildinterpretation besteht besonders darin, "den Schüler zur selbständigen Betrachtung, zum Erschliessen des geographisch Wesentlichen und Typischen" zu erziehen, wozu "eine geduldige und fortgesetzte Uebung" unbedingt notwendig ist $(3)$.

Weizenanbau in der kanadischen Prärie

Ziel der Lektion:

Die Schüler (8.-9.Schuljahr) sollen Einsicht gewinnen in den Lebens- und Wirtschaftsraum der kanadischen Prärie.

Unterrichtsverlauf:

Einstieg:

Die Lektion begann mit einem Bild, das Indianer auf der Büffeljagd zeigt, womit das Interesse und die Neugier für das Kommende geweckt werden sollte. Der Lehrer gab dazu folgenden Kommentar ab: "Bei ihrem Vorstoss ins Innere des nordamerikanischen Kontinentes entdeckten die weissen Pioniere riesige Grasländer, auf denen Tausende von Büffeln lebten, die von den Indianern, den Herren des Landes, gejagt wurden. Später, als sich dann landsuchende Siedler in diesem Gebiet niederzulassen begannen, wurden die Büffel fast völlig ausgerottet, und die Indianer zum Verlassen ihrer Jagdgrüinde gezwungen."

Anschliessend wurde die Klasse gefragt, ob andere Bezeichnungen für diese Grasländer, beziehungs-

Prof. H. Inhelder, Geographielehrer, Buchzelgstrasse 32, 8053 Zürich. 
weise Büffel, bekannt seien. Spontan kamen die Antworten "Prärie" und "Bison". Die Herkunft des ersten Begriffs vom französischen Wort "la prairie = Wiese" war allerdings unbekannt.

Bildbesprechung:

Als Kontrast zum ersten Bild wurde dann das Bild "Weizengebiet bei Riceton, Saskatchewan" mit folgender Bemerkung gezeigt: "Wer heute die Präriegebiete bereist, findet die Landschaft auf weite Strecken völlig verändert vor."

Die Schüler wurden daraufhin aufgefordert, hervorstechende Einzelheiten des Bildes zu nennen. Die Antworten, die der Lehrer fortlaufend in Stichworten an die Tafel schrieb, ergaben folgendes Resultat:

"flaches Land" - "eintönige Ebene" - "riesige Getreidefelder" - "Ebene erscheint gestreift" "verstreute Farmen" - "schnurgerade Strasse" "Bäume nur bei den Farmen" - "Kleine Siedlung mit Silos" - "Eisenbahnlinie" - "Himmel wolkenlos" - "fruchtbarer Boden" - "Ansammlung von Maschinen" - "Grasland bei den Farmen" - "helle und dunkle Felder" - "fast menschenleer" - "trockene Landschaft".

Diese Phase des Unterrichtes, das Umsetzen des Gesehenen in Worte ist ausserordentlich wichtig, weil dadurch der Bildeindruck besser im Gedächtnis verankert wird. Der Lehrer muss deshalb darauf achten, dass die Antworten, entsprechend der Altersstufe, einfach und klar formuliert werden. Ferner können bei dieser Gelegenheit neue Bezeichnungen eingefüht oder ungenaue Benennungen korrigiert werden. So wurden im vorliegenden Fall die Begriffe "Monokultur" und "strip-farming" eingeführt und die Bezeichnungen Höfe und Schuppen durch Farmen und Silos ersetzt.

Stillarbeit mit anschliessender Diskussion: Der nächste Unterrichtsschritt verlangte von den Schiilern das Herausfinden von Zusammenhängen zwischen den gemachten, an der Tafel stehenden Beobachtungen. Die Ergebnisse mussten auf einem Blatt notiert werden. Häufig waren Zusammenhänge zwischen zwei Beobachtungen. Es gab aber auch verschiedene Dreierkombinationen. Einige davon seien hier erwähnt:

"Flaches Land - riesige Getreidefelder - verstreute Farmen." "Riesige Getreidefelder kleine Siedlung mit Silos - Eisenbahnlinie." "Eintönige Ebene - schnurgerade Strassen - fast menschenleer." "Wolkenloser Himmel - trockene Landschaft - riesige Getreidefelder." "Riesige Getreidefelder - fast menschenleer - Ansammlung von Maschinen." "Eintönige Ebene - verstreute Farmen - Bäume nur bei den Farmen." "Flaches Land - helle und dunkle Felder - Ebene erscheint gestreift."

Die angeführten Beispiele zeigen deutlich, dass im Anschluss an das reine Sehen, an das Betrachten der einzelnen Bildgegenstände, Denkprozesse und Ueberlegungen einsetzten, die auf früher erworbenen Kenntnissen beruhen. Einzelne Verknüpfungen erforderten selbstverständlich eine genauere Auslegung durch den Schüler und ein Nachfragen durch den Lehrer. Dieses Frag- und Antwortspiel ermöglichte es auch den schwächeren Schuilern, den Gedankengängen ihrer Klassen- kameraden zu folgen. Zudem bot sich hier Gelegenheit, auf weitere Probleme einzugehen. In Verbindung mit der erwähnten "Ansammlung von Maschinen" schilderte der Lehrer, wie heute, dank der Mechanisierung und Rationalisierung, die Feldarbeiten von wenigen Leuten besorgt werden können. Automatisch kamen dabei weitere Themen, wie Landflucht und Zunahme der Hofgrösse zur Sprache. Die Besprechung der Vor- und Nachteile der Monokultur beanspruchte ziemlich viel Zeit. Erstaunlich war, dass der Begriff "Brache" verschiedenen Schülern überhaupt nichts bedeutete. Die Aussage "Bäume nur bei den Farmen" benutzte der Lehrer, um die Aufmerksamkeit der Klasse auf die an der Wand hängende physische Karte Nordamerikas zu lenken. Die Offenheit der zentralen Tieflandzone für Winde aus Norden und Suiden wurde von allen sehr rasch erkannt.

\section{Atlasarbeit:}

Anhand verschiedener Karten stellten die Schüler zunächst die Ausdehnung des Präriegebietes fest. Die Ungenauigkeit der Bezeichnung "Prärieprovinzen" für Manitoba, Saskatchewan und Alberta ergab sich dabei fast von selbst. Für die gemachte Feststellung "fast menschenleer" fand sich auf der Volksdichtekarte eine Bestätigung. Dagegen gab es im Atlas nirgends einen Hinweis, der die Behauptung "fruchtbarer Boden" hätte belegen können. Der Lehrer war daher gezwungen, diese Lücke mit einigen Erläuterungen zu schliessen:

Erfolgreich war dagegen die Arbeit mit den Klimakarten. So fanden die Schüler auf der Karte der Nièderschläge die Bemerkung "vorwiegend Regen im Frühsommer" und stellten im übrigen fest, dass die Prärien ein niederschlagsarmes Gebiet sind. Das Studium der Isothermenkarten zeigte ferner tiefe Januar- und relativ hohe Julitemperaturen. Der Lehrer wies des weiteren auf die Bedeutung dieser Angaben für das Wachstum, die Reife und die Ernte des Weizens hin. Auf die Frage, um was für eine Weizenart es sich demnach handeln müsse, lautete die Antwort einstimmig: "Sommerweizen". Dank der Frage eines Schülers konnten noch einige Bemerkungen zur Züchtung rasch reifender Weizensorten und zur Ausdehnung des Weizenanbaugebietes nach Norden eingeflochten werden. Zwangsläufig kam man damit auch auf die Anbaugrenze im Westen und die damit verbundenen Probleme zu sprechen.

Zuletzt wurden auf den Atlaskarten noch die verschiedenen Transportwege des Weizens und die wichtigsten Ausfuhrhäfen aufgesucht. Probleme des Weizentransportes und -exportes kamen aber aus Zeitmangel nicht mehr zur Sprache.

Soweit es möglich war, hielt der Lehrer auch in diesem Unterrichtsabschnitt die Schüler stets zu eigener Kartenarbeit an. Natürlich waren immer wieder lenkende Fragestellungen notwendig, um ans gewünschte Ziel zu gelangen. Aber auch hier gilt: was selbst erarbeitet wird, bleibt besser haften.

Zusammenfassung:

Während der Lektion erfolgte kein Hefteintrag. Die Schuiler konnten sich somit voll auf die gestellten Aufgaben und das Unterrichtsgespräch 
konzentrieren. Dafür erhielt jeder am Ende der Stunde ein vervielfältigtes Blatt mit einer vereinfachten Darstellung des besprochenen Bildes in Form einer Strichzeichnung und dem folgenden Text:

- Mit dem Wort "Prärie" (frz. la prairie = Wiese) bezeichnet man die weiten, baumlosen Grasländer Nordamerikas. Durch den Eingriff des Menschen wurden grosse Teile dieser Naturlandschaft in Kulturland umgewandelt.

- Der fruchtbare Boden und die herrschenden $\mathrm{Kl}$ imaverhältnisse (Regen im Frühsommer zur Wachstumszeit, sowie Wärme und Trockenheit zur Reife- und Erntezeit) begünstigen vor allem im östlichen Teil den Anbau von Sommerweizen.

- Die einzelnen Farmen liegen inmitten riesiger Felder im Windschutz von kleineren Baumgruppen. Das flache Land gestattet den Einsatz gewaltiger und leistungsfähiger landwirtschaftlicher Spezialmaschinen, für deren Bedienung nur wenige Leute benötigt werden (Landflucht, geringe Bevölkerungsdichte).

- Der geerntete Weizen wird vom Feld direkt zu den Silos der nächsten Bahnstation gebracht. Lange Güterzuige befördern ihn dann zu den Häfen am Oberen See (Fort William, Port Arthur), nach Vancouver oder nach Churchill an der Hudsonbay.

- Die Monokultur ist risikoreich (Missernten, Ueberproduktion, Preisschwankungen usw.). Viele Farmer gehen daher immer mehr zu einer vielseitigeren Landwirtschaft (mixed farming) über.

Hausaufgabe:

Auf die nächste Stunde mussten die wichtigsten Bildgegenstände auf der Strichzeichnung numeriert und in einer Legende zusammengefasst werden.

Im Zusammenhang mit dem Weizenanbau in der kanadischen Prärie könnte selbstverständlich noch der eine oder andere Punkt erwähnt werden. Da aber das nebenstehende Bild im Mittelpunkt der Stunde stand, konzentrierte sich das Unterrichtsgespräch nur auf Fragen, die mehr oder weniger direkt mit ihm in Verbindung gebracht werden konnten. Diese Beschränkung hatte den Vorteil, dass der Schüler nie die Uebersicht verlor. Natiurlich ist jede Lektion variabel und kann entsprechend den Vorkenntnissen der Klasse und den Absichten des Lehrers ausgebaut, verkürt oder verschiedenartig abgewandelt werden. Dies ist ja auch der Reiz des Unterrichtens und verhindert einen Ablauf in festgefahrenen Bahnen. Wer sich Zeit nimmt, $a b$ und zu eine eingehendere Bildbetrachtung in seine Lektionen einzubauen, wird sehr bald erkennen, dass man dabei selbst immer wieder neue Denkanstösse erhält und oft ungewollt vom Lehrenden zum Lernenden wird.

\section{Literatur}

1 Ebling, Hans: Anschauen, behandeln, begreifen, S. 8, Hannover 1964.

2 Fick, Karl E.: Bildbetrachtung im Erdkundeunterricht, S. 49, in Marienauer Chronik, H.20, 1967.

3 Heyn, Erich: Lehren und Lernen im Geographieunterricht, S. 60, Verlag Schöningh, Paderborn 1973.

4 Ritter, Gert: Das Lichtbild im Unterricht, S. 38 - 39, Der Erdkundeunterricht, Heft 12, E. Klett-Verlag, Stuttgart 1972.

5 Schmidt, Alois: Der Erdkundeunterricht, S. 267, Verlag Julius Klinkhardt, Bad Heilbrunn/OBB., 1972.

6 Wocke, M.F.: Film und Bild im geographischen Sachunterricht, Hermann Schroedel Verlag KG, Hannover, 1973.

\section{Literaturbesprechungen}

GRENZEBACH Klaus (Hrsg.): Entwicklung der Landnutzung in den Tropen und ihre Auswirkungen, Giessener Beiträge zur Entwicklungsforschung, Schriftenreihe des Tropeninstituts der Gustav Liebig Universität Giessen, Reihe I (Symposien), Band 2, Tropeninsitut Giessen 1976, 121 S., 11 Beiträge mit diversen Fig., Tab., Photographien und Profilen.

Im Mai 1976 wurden die in diesem Band vereinigten Beiträge als Vorträge anlässlich des zweiten Symposiums des Tropeninstituts der Universität Giessen einem interdisziplinären $\mathrm{Au}$ ditorium dargeboten.
In den auf Fallstudien bezogenen Vorträgen wurden Prozesse der Landnutzung in den Tropen aus folgenden fachlichen Perspektiven analysiert: Geographie der Tropen, Pflanzenbau und Pflanzenzuichtung, Bodenkunde und Bodenerhaltung, Phytopathologie und Angewandte Entomologie, Tropische Veterinärmedizin sowie Tierzucht und Tierernährung.

Vier Beiträge sind für den Geographen von besonderem Interesse: H.ROHDENBURG untersucht in Suid- und Nordnigeria den Ursachenkomplex Landnutzung - Klima in seiner Bedeutung für die Bodenerosion. Es werden Beispiele für vorzeit- 